\title{
Evaluación de las pérdidas de arroz en la mini cosechadora LOVOL AF-100 (Serie 0) en la Granja Agrícola Arrocera "Bartolomé Masó Márquez"
}

\author{
Dr.C. Benjamín Gabriel Gaskins Espinosa,
}

Universidad de Granma. Facultad de Ciencias Técnicas.

Bayamo, Granma, Cuba

Dr.C. Idalberto Macías Socarrás, Universidad Estatal de la Península de Santa Elena (UPSE).

Facultad de Ciencias Agrarias. Santa Elena, Ecuador

M.Sc. Ernesto Reyna Pompa,

Universidad de Granma. Facultad de Ciencias Técnicas.

Bayamo, Granma, Cuba

M.Sc. Miguel Bolívar Zamorano Reyes,

Facultad de Ingeniería Agropecuaria. Carrera de Ing. Agropecuaria.

Universidad Laica Eloy Alfaro de Manabí

M.Sc. Antonio Luis Barrera Amat,

Escuela Superior Politécnica del Chimborazo.

Extensión Norte Amazónica. ESPOCH. Ecuador

Doi: 10.19044/esj.2018.v14n33p35 URL:http://dx.doi.org/10.19044/esj.2018.v14n33p35

\begin{abstract}
The investigation, refers to the Agricultural Farm of Rice "Bartolome Maso Marquez", located in the municipality of Yara, province of Granma, is dedicated to the production of rice, the objective is based on the total losses of the grains and the system of the machine, The quality of the harvested product is also evaluated, using the LOVOL-AF 100 (Series 0) mini harvester. The combined losses were 5.98\%; I found above the parameters set by the manufacturer of 5\%; There are significant differences between the losses in the different parts of the combination, with a better performance in the $1.62 \%$ sieve, followed by agitation with $2.03 \%$, in the pre-harvest the losses were $3.5 \%$ much higher than those established, of $1 \%$.; The content of impurities in the harvested mass reached $13 \%$, higher than the established in $30 \%$, the concept of losses of the combine in total reached $23920 \mathrm{t}$ of clean rice, which represents the consumption of rice of 322222 people in one year.
\end{abstract}

Keywords: Losses, sieve, shaker, pre-harvest and platform 


\section{Resumen}

La investigación, se desarrolló en la Granja Agrícola Arrocera "Bartolomé Masó Márquez" ubicada en el municipio Yara, provincia Granma, dedicada a la producción de arroz, el objetivo fue evaluar las pérdidas totales de granos y por sistema de la máquina, igualmente se evaluó la calidad del producto cosechado, utilizando la minicosechadora LOVOLAF 100 (Series 0). Las pérdidas totales de la cosechadora fueron de 5,98 \%; encontrándose por encima de los parámetros establecidos por el fabricante de $5 \%$; existen diferencias significativas entre las pérdidas en las diferentes partes de la combinada, con mejor comportamiento en la zaranda 1,62 \%, seguido del sacudidor con $2,03 \%$, en la precosecha las pérdidas fueron de 3,5 $\%$ muy superiores a los establecidos, de $1 \%$; los contenidos de impurezas en la masa cosechada alcanzaron un $13 \%$, superior al establecido en un $30 \%$, por concepto de pérdidas de la cosechadora en total se alcanzaron $23920 \mathrm{t}$ de arroz limpio, lo que representa el arroz consumo de 322222 persona en un año.

Palabras Claves: Pérdidas, zaranda, precosecha y plataforma

\section{Introducción}

La producción de arroz en Cuba presenta un alto grado de mecanización. La maquinaria que participa en la cosecha de arroz, pertenece a una determinada Empresa Agroindustrial de Granos (EAIG) y se agrupa en complejos mecanizados, que a su vez conforman los eslabones productivos de la cosecha, aspecto que permite lograr una mejor atención a los problemas organizativos, técnicos y tecnológicos que surgen durante el proceso productivo. (Morejón, 2015).

En Cuba se han efectuado investigaciones dirigidas al incremento de la efectividad de la cosecha mecanizada del arroz, sin embargo, las mismas han estado dirigidas a la optimización de aspectos explotativos del proceso, no incluyendo el ajuste de los parámetros de regulación de los órganos de trabajo dirigido a la disminución de las pérdidas. Asimismo, no han sido reportados estudios que brinden datos sobre las pérdidas ocasionadas durante la cosecha mecanizada del grano, así como del peso relativo de los diferentes órganos de trabajo de las máquinas en la producción de dichas pérdidas. (Miranda et al., 2006; Matos et al., 2010; Morejón, 2015)

Varias son las investigaciones que se han realizado en el estudio de las pérdidas de cosecha que abarca numerosos aspectos, según el INTA, (2015) las operaciones que se suceden a lo largo del proceso de cosecha, son las causas que provocan esas pérdidas, como son los depredadores y parásitos de los productos, sin hablar de las condiciones físicas, técnicas, económicas 
y otras, que favorecen la acción de los agentes de deterioro, y en consecuencia, la agravación de las pérdidas. Según las opciones y los objetivos de estos autores particularmente de las pérdidas se dividen en las causas primarias, las causas secundarias, los lugares y momentos de las pérdidas.

Actualmente, los índices de pérdida de granos admisibles en las cosechadoras comprende hasta el 5\%, sin embargo se obtienen cifras que duplican este valor. Provocadas fundamentalmente por mal manejo de las máquinas y cuestiones agro-técnicas del arroz, estas cosechadoras poseen censores con indicadores de barras que permiten determinar si está existiendo pérdida de granos por limpieza y trilla (la cola), y así tomar acciones para corregir el problema, sin embargo el dato cuantificado en unidad de medida aún no se conoce, lo que limita a hacer análisis pos-cosecha para revertir de forma positiva la perdida en el campo. (Pozzolo y Pirovani, 1990)

En las zonas tropicales, durante la estación seca, el arroz está pronto para ser cosechado, por lo general 25 a 35 días después de la floración total. Casi siempre, en esta etapa, el 85 - 90 por ciento de las panojas tienen color amarillo a amarillo-dorado. Cualquier demora en la cosecha resulta en pérdidas causadas por roedores, aves, vuelco y desgrane. Si las panojas están húmedas debido a las lluvias o al agua estancada, las semillas pueden germinar en la espiga causando importantes pérdidas en cantidad y calidad. (Chaudhary et al., 2003).

Partiendo de lo antes expuesto a la serie 0 de la cosechadora LOVOL AF 100, diseñada en el CEDEMA y fabricada en la República Popular China, es necesario realizarle la prueba de desarrollo con la tarea técnica aprobada por el Instituto de Investigaciones Agrícolas de Cuba (IAgric) a solicitud Dirección General del Grupo Empresarial de la Industria Sidero Mecánica (GESIME) en febrero del 2017.

A partir de lo anterior se define como objetivo: Evaluar las pérdidas de cosecha del arroz de la mini cosechadora de arroz LOVOL AF-100 bajo las condiciones de la Granja Agrícola Arrocera "Bartolomé Masó Márquez" de la UEB Arrocero "Fernando Echenique".

\section{Materiales y métodos}

La investigación, se desarrolló en la en la Granja Agrícola Arrocera "Bartolomé Masó Márquez" de la UEB "Fernando Echenique" municipio Yara, provincia Granma, la misma se ubica en los 20 16'36" latitud norte y 76 56'49" latitud oeste, con una extensión de 5529 ha dedicadas a la producción de arroz, limitada por el Norte con la carretera Yara-Manzanillo, por el sur con el Consejo Popular Mateo Romas, por el este con el Consejo Popular José Martí y por el oeste con el Río Jibacoa, situada a 10 msnm. En un vertisol con topografía llana, buena nivelación y drenaje, con sistema 
semi-ingeniero que permite buen acceso al lote arrocero. La temperatura osciló entre 27 y $30^{\circ} \mathrm{C}$ y una humedad relativa promedio del $78 \%$, con la variedad de arroz LP-5, con un porcentaje de maduración entre 80 a $95 \%$ en campos con rendimiento entre 3,5 a 4,0 $\mathrm{t} \mathrm{ha}^{-1}$.

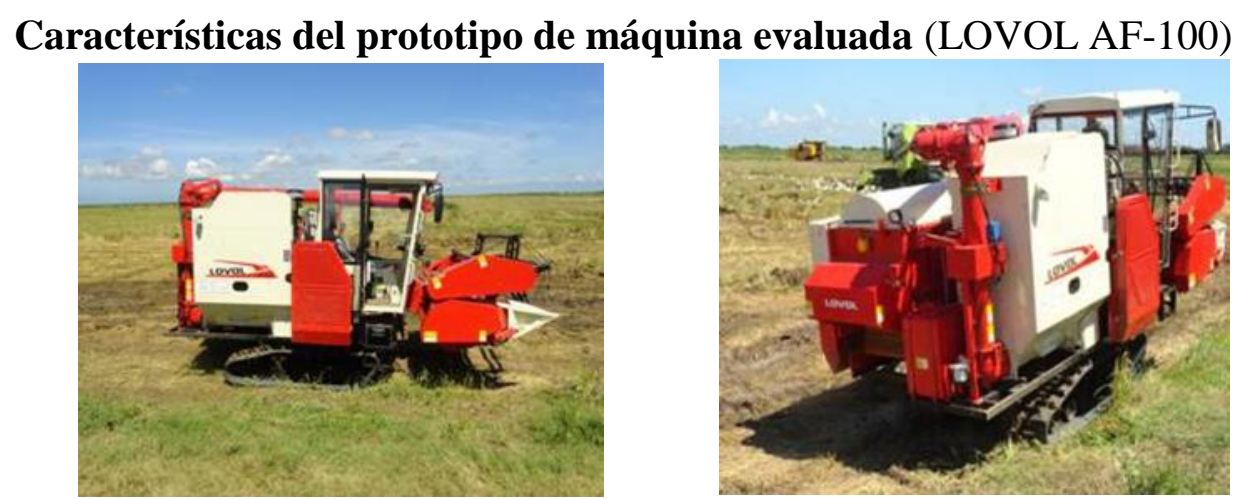

Figura 1.- Prototipo de la Minicosechadora autopropulsada:

Las pruebas se le realizaron al prototipo de la Minicosechadora autopropulsada:

- Marca: LOVOL

- Modelo: AF 100

- País de fabricación: República Popular China

- Cantidad de máquinas aprobadas para la prueba: 1 prototipo modificado (Serie 0).

- Fecha de realización de la prueba: Periodo comprendido entre septiembre 2017 a enero 2018.

En todos los casos, para expresar los valores obtenidos en $\mathrm{kg}$ de pérdidas por hectárea, todas las observaciones parten de un pre-experimento con 25 muestra a las cuales se le determina el tamaño de muestra necesario para que sean representativos para obtener datos más confiables. Es importante destacar que además se realizó un diagnóstico de cómo se realizó la trilla para observar la existencia de grano sin trillar, grado de limpieza en la tolva, grano partido y materias extrañas.

Se evaluó la tecnología de cosecha en cuatro parcelas o terrazas (T1, T2, T3, T4) con condiciones agrotécnicas distintas, de (300 m x $150 \mathrm{~m})$ con un área de 4,5 ha. Ver figura 2 y 3. 


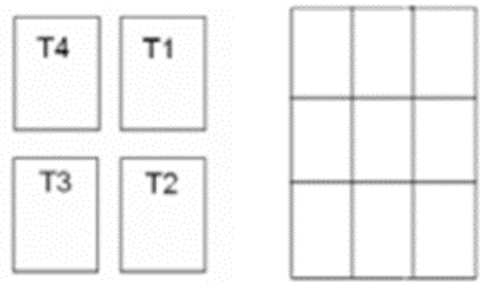

Figura 2.- Esquema de la ubicación de las terrazas y el área experimental.
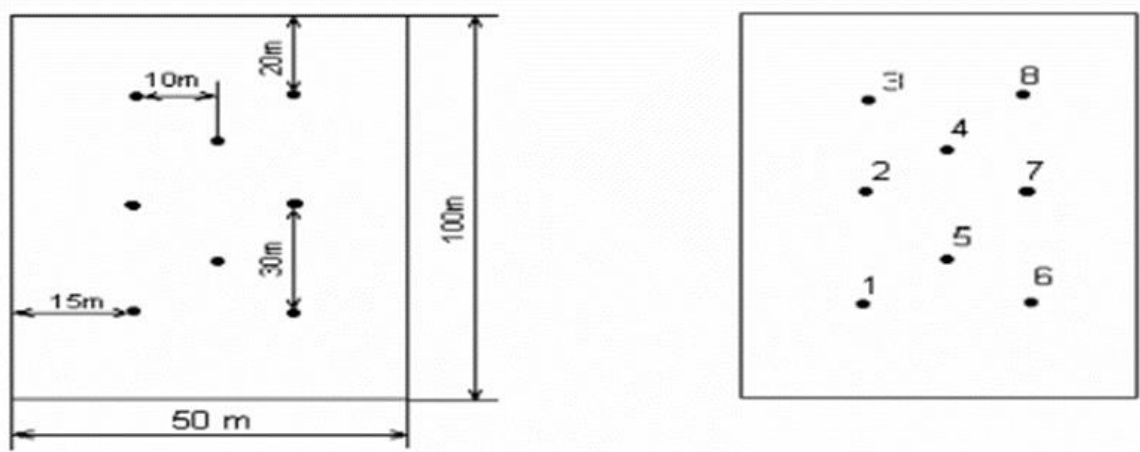

Figura 3.- Croquis y numeración de los puntos de muestreo.

Para llevar a cabo la experimentación se utilizó el mismo método en cada una de las terrazas y en cada muestreo, que se relaciona a continuación.

Se utiliza un diseño completamente aleatorizado, se dejan $10 \mathrm{~m}$ entre parcela y entre la guardarraya y la parcela objeto de estudio para evitar el efecto de borde. El tamaño de la muestra para cada una de las variantes analizadas se determina por el programa estadístico Sample, a partir de un preexperimento con un tamaño de muestra de 25 , procesándose estadísticamente los resultados obtenidos y determinándose los estadígrafos fundamentales (media, desviación estándar y el error), para un error de la media menor del $5 \%$ y una probabilidad del 95\%, (Carballosa et al, 2014) El cálculo se efectuara por la expresión (1):

$n=\frac{\sigma^{2} \mathrm{t}^{2}}{\Delta}$

donde:

n: tamaño de la muestra requerido;

s: desviación media cuadrática obtenida en el pre-experimento;

$\Delta$ : error máximo de la media deseado;

t: criterio de Student para el tamaño de la muestra del pre-experimento y el nivel de probabilidad o significación declarado (95\%). 


\section{Metodología para determinar las pérdidas precosecha}

Para llevar a cabo la experimentación se utilizó el mismo método en cada una de las terrazas y en cada muestreo, que se relaciona a continuación. Antes de evaluar la pérdida de granos de cualquier mecanismo de la cosechadora es necesario determinar los granos caídos en el suelo antes del inicio de la cosecha y así restar esta pérdida a la provocada por la máquina. Según la metodología propuesta por el Ministerio de la Agricultura en una zona representativa del lote se colocan 4 marcos de $0.25 \mathrm{~m}^{2}$ cada uno, equivalentes a $1 \mathrm{~m}^{2}$, se cuentan los granos, panojas sueltas y las que no son recolectadas por el cabezal consideradas en general como las que se encuentran por debajo de la altura de corte fijada. (Quesada y Salinas, 2017). (Ver Figura 4).

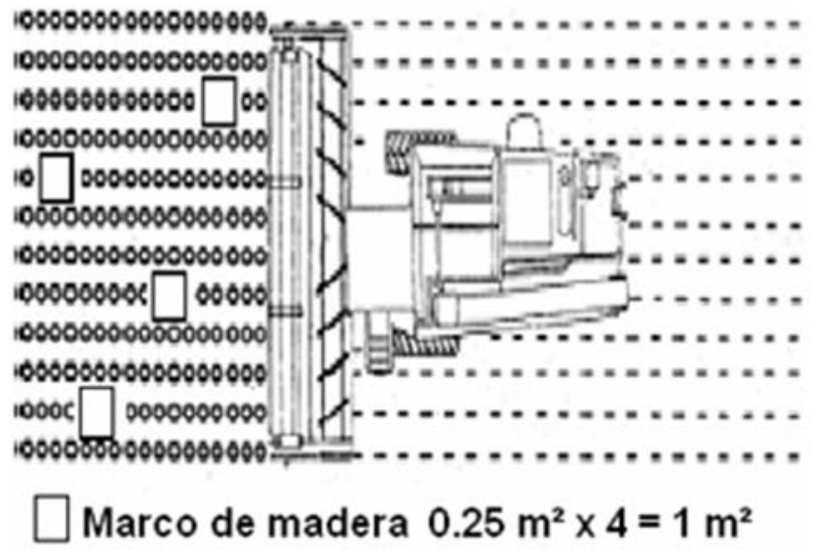

Figura 4. Colocación de los marcos para determinar las muestras de pérdidas de precosecha (Fuente INTA, 2006)

Para determinar la pérdida de precosecha en $\mathrm{kg} \cdot \mathrm{ha}^{-1}$, se juntan los granos sueltos y lo obtenido de las panojas desgranadas, teniendo en cuenta que, para la variedad LP-5, 250 granos representan $100 \mathrm{~kg}$ de pérdida por hectárea. En la experimentación se realiza el conteo de los granos, sin embargo, es conveniente tomar un recipiente transparente de aproximadamente $150 \mathrm{~cm}^{3}$ y hacer una marca luego de echar los 250 granos. De esta forma, en cada oportunidad en que se necesite evaluar pérdidas, bastará con comparar la cantidad de granos perdidos con la marca efectuada en el frasco. (Quesada y Salinas, 2017). (Ver figura 5).

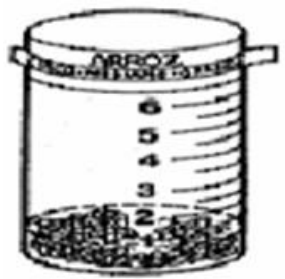

Figura 5. Frasco para recolectar los granos de arroz (Fuente INTA, 2006) 
Se debe tener cuidado al entrar al cultivo debido a que generalmente existe una importante masa vegetal con panojas entrelazadas que facilita el desgranado por efecto de la persona. Esto es particularmente importante con humedades del grano por debajo del 17 por ciento. Las pérdidas precosecha se evaluaron en terreno en seco. (Quesada y Salinas, 2017). (Ver Figura 6).

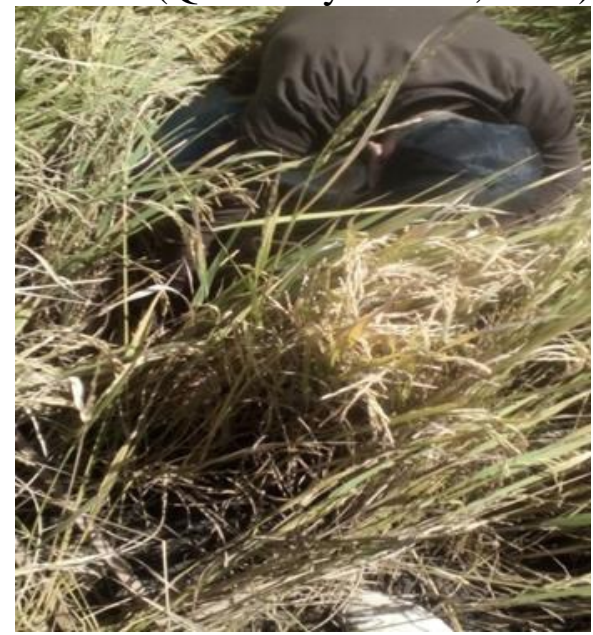

Figura 6. Recolección de los granos de precosecha

\section{Metodología para determinar las pérdidas por la cola}

Estas pérdidas se dividen en dos: las originadas en el cabezal y las provocadas por la cola, que a su vez se dividen en pérdidas por zaranda y saca pajas. Las pérdidas de arroz por la cola se determinan arrojando los 4 marcos ciegos después del paso del cabezal y antes que caiga el material por la cola, uno por debajo del cajón de zarandas (zona central) y los 3 restantes, en el área que abarca el cabezal. (INTA, 2006).

En esta experimentación también se toma uno de los marcos, se forra con un saco de nylon y se coloca exactamente debajo de las zarandas primero y luego de recolectar las muestras se ponen debajo de los saca pajas, de forma individual y con cuidado para que no se mezclen los restos obtenidos, luego se procede a realizar la misma operación de conteo para ser más fiable los resultados. Una vez que pasa la cosechadora se procede a separar del material recolectado, primeramente se extrae todo el material grueso (paja y tallos), quedando en la bandeja los granos completos y granos vanos, pero como la separación manual de éstos es una tarea que demanda tiempo y es frecuente encontrar gran cantidad de vanos, se colocan todos los granos contenidos en la bandeja (enteros y vanos) en una botella plástica con el extremo inferior cortado (la base) y la tapa roscada sujetando a la botella de modo que el pico quede hacia abajo, a continuación se coloca agua hasta la mitad de la botella y se verifica que los vanos floten en la superficie, mientras que los demás se mantendrán inmersos en el fondo (contra la tapa), luego se vuelca 
cuidadosamente el agua con los vanos, para después desenroscar la tapa y extraer los granos llenos logrando, de este modo, una correcta separación, posteriormente se cuentan de la manera ya explicada en pérdidas de precosecha.

\section{Metodología para determinar las pérdidas en la plataforma de corte}

Se recogen todos los granos y panojas sueltas que quedan por debajo de los cuatro aros ciegos, obteniendo así una muestra de $1 \mathrm{~m}^{2}$ que incluye la pérdida de cabezal más las de precosecha que fueron restadas.

Durante la toma de muestras se realiza este método, pero para ser más fiel en los resultados, se desplaza la cosechadora 50 metros cosechando y se para repentinamente, luego la máquina se traslada hacia atrás dejando al descubierto el área cosechada, se miden 2 metros a contar del primer cultivo que no fue cortado y se colocan los aros ciegos a lo ancho de la plataforma.

\section{Análisis y discusión de los resultados}

En este capítulo se analiza los resultados obtenidos en la investigación para lo cual se realizó un análisis de varianza de clasificación simple con la prueba de mínima diferencia significativa (LSD) como variable independiente el porcentaje de pérdida y los factores los lugares donde se producen las pérdidas (plataforma, zaranda y sacudidor) y la de precosecha.

Tabla 1: Pérdidas de cosecha

\begin{tabular}{lccccc}
\hline \multicolumn{1}{c}{ Fuente } & $\begin{array}{c}\text { Media } \\
\text { \% }\end{array}$ & Varianza & $\begin{array}{c}\text { Desviación } \\
\text { típica }\end{array}$ & $\begin{array}{c}\text { Error } \\
\text { estándar }\end{array}$ & P-valor \\
\hline Precosecha & $3,5^{\mathrm{a}}$ & 0,22 & 1,10 & 0,21 & \\
Plataforma & $2,35^{\mathrm{b}}$ & 1,69 & 1,64 & 0,32 & 0,032 \\
Zaranda & $1,6^{\mathrm{c}}$ & 1,36 & 1,16 & 0,22 & \\
Sacudidor & $2,03^{\mathrm{d}}$ & 0,67 & 0,82 & 0,16 & \\
\hline
\end{tabular}

Como se observa en la tabla 1, existe diferencia significativa entre los factores analizados teniendo un mejor porcentaje la zaranda con 1,62\% y un valor mayor en la plataforma de corte, en este caso este valor se incrementó por el golpe del molinete sobre las espigas al grado de maduración el cual era superior al 95\%, según las observaciones del laboratorio de la industria y con lo cual aumenta las pérdidas, estos valores son similares a los obtenidos en las evaluaciones realizada por los especialista IIMA de la provincia de Holguín, por ser esta una máquina de nueva introducción de la agricultura arrocera.

En cuanto a las pérdidas de la plataforma los resultados obtenidos de 2,35\%, son muy superiores a los obtenidos por Torres-Sotolongo y Martínez, (2017) cuando evaluaron cosechadora CLAAS CROP TIGER-30 en la variedad "Reforma".

Obteniendo un valor total de las pérdidas de 5,98 \% muy superior a los obtenidos por Torres-Sotolongo y Martínez (2017) cuando evaluaron la 
cosechadora CLAAS CROP TIGER-30 y del 3\% de acuerdo a la Normas Brasileñas, (1987), y similares a los de las pérdidas reportadas por otros autores como Platero, (1992); Andrews et al.,(1993); Alonso et al., (1999); Aristizabal et al., (1999); Ferreira et al., (2001), Pozzolo et al., (2006) y Ramos, (2018).

Si se tiene en cuenta que Cuba produce aproximadamente unas 400 000 t de arroz limpio, lo que equivale a 666 666,66 t de arroz en cáscara, teniendo en cuenta el valor de las pérdidas, alcanzarían 39 866,66 t de arroz en cáscara que se pierden, conociendo que en el proceso industrial el rendimiento es de alrededor del $60 \%$, entonces se dejarían de producir 23920 t de arroz limpio, por lo que el país debería importar esa cantidad adicional, lo que representa el arroz consumo de 322222 persona en un año.

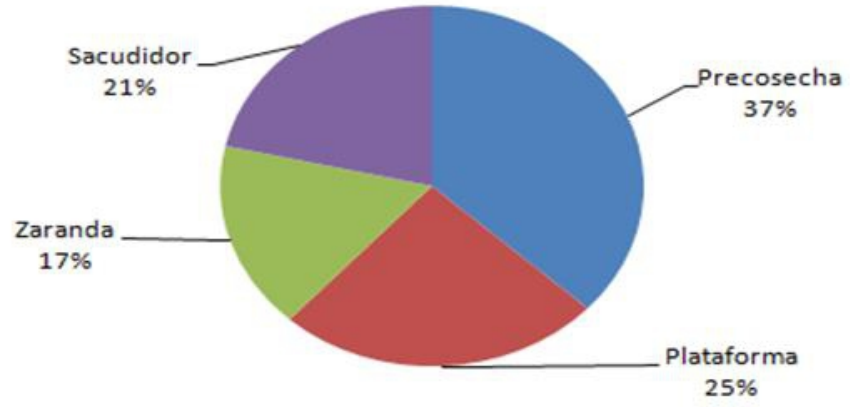

Figura 7. Porcentaje de pérdidas

En la figura 7, se observa que la mayor cantidad de pérdidas es de la precosecha esto se debe al porcentaje de maduración que era del $95 \%$, según Chaudhary et al., (2003) plantean que el 85 - $90 \%$ de las panojas tienen color amarillo a amarillo-dorado. Cualquier demora en la cosecha resulta en pérdidas causadas por roedores, aves, vuelco y desgrane. Si las panojas están húmedas debido a las lluvias o al agua estancada, las semillas pueden germinar en la espiga causando importantes pérdidas en cantidad y calidad. Las pérdidas achacables a la plataforma de corte es del $25 \%$, siendo inferiores a lo obtenido por autores como Da Silva et al., (1983); Aristizabal et al., (1999), quienes reportan pérdidas de granos debido a una deficiente regulación del molinete en un rango entre 33 y $73 \%$ de las pérdidas totales y Torres-Sotolongo y Martínez (2017) con un 42,44 \% en la variedad "Reforma" con la cosechadora CLAAS CROP TIGER-30.

Al realizar una valoración de la composición de la masa recolectada por la máquina y teniendo en cuenta los parámetros que establece la industria (NRAG-910, 1988) de materia extraña en la masa cosechada como son:

Vegetales y tallos: $2 \%$; semillas indeseables: $0,2 \%$; tierras y piedras: 0,4\%; cáscara, paja, vanos, semi vanos y pastosos: $4 \%$; granos pelados partidos y cáscara partido: 1,2\%; granos lechosos verdes: 2,2\%. 
Es decir que el total de materia extraña no debe sobrepasar el $10 \%$, como puede observarse en la tabla 2 (figura 8), se obtiene valores superiores a $13,21 \%$, un 3,21 por ciento mayor que lo establecido por la industria, estos valores son superiores a los obtenidos por Miranda et al., (2010) de 8,3 y 9,6 $\%$, con cosechadora NEW HOLLAND L521 y Miranda et al., (2006), con las cosechadoras NEW HOLLAND L520 e Ideal International 9075.

Por otro lado, cuando se realizó la caracterización de las materias extraña se puedo comprobar que los mayores porcentajes eran (vegetales y tallos; semillas indeseables; tierras y piedras) y en menor cuantía los granos pelados entero y partido, un aspecto importante en la evaluación de esta máquina es que los granos sin daños alcanzan el 98,71 \%, este valor es inferior en 0,39\% a los obtenidos por (Quesada y Salinas, 2017).

Tabla 2.- Porcentaje de materias extrañas en la masa cosechada

\begin{tabular}{lccccc}
\hline \multicolumn{1}{c}{ Fuente } & $\begin{array}{c}\text { Media } \\
\text { \% }\end{array}$ & Varianza & $\begin{array}{c}\text { Desviación } \\
\text { típica }\end{array}$ & $\begin{array}{c}\text { Error } \\
\text { estándar }\end{array}$ & P-valor \\
\hline $\begin{array}{l}\text { Granos entero } \\
\text { pelados }\end{array}$ & $0,52^{\mathrm{a}}$ & 0,005 & 0,072 & 0,014 & \\
$\begin{array}{l}\text { Granos partido } \\
\text { pelados }\end{array}$ & $0,77^{\mathrm{b}}$ & 0,002 & 0,044 & 0,008 & \\
Granos sin daños & $98,71^{\mathrm{c}}$ & 0,238 & 0,488 & 0,097 & 0,002 \\
Granos verdes & $2,82^{\mathrm{d}}$ & 0,059 & 0,243 & 0,048 & \\
Materias extrañas & $13,21^{\mathrm{e}}$ & 0,282 & 0,088 & 0,091 & \\
\hline
\end{tabular}

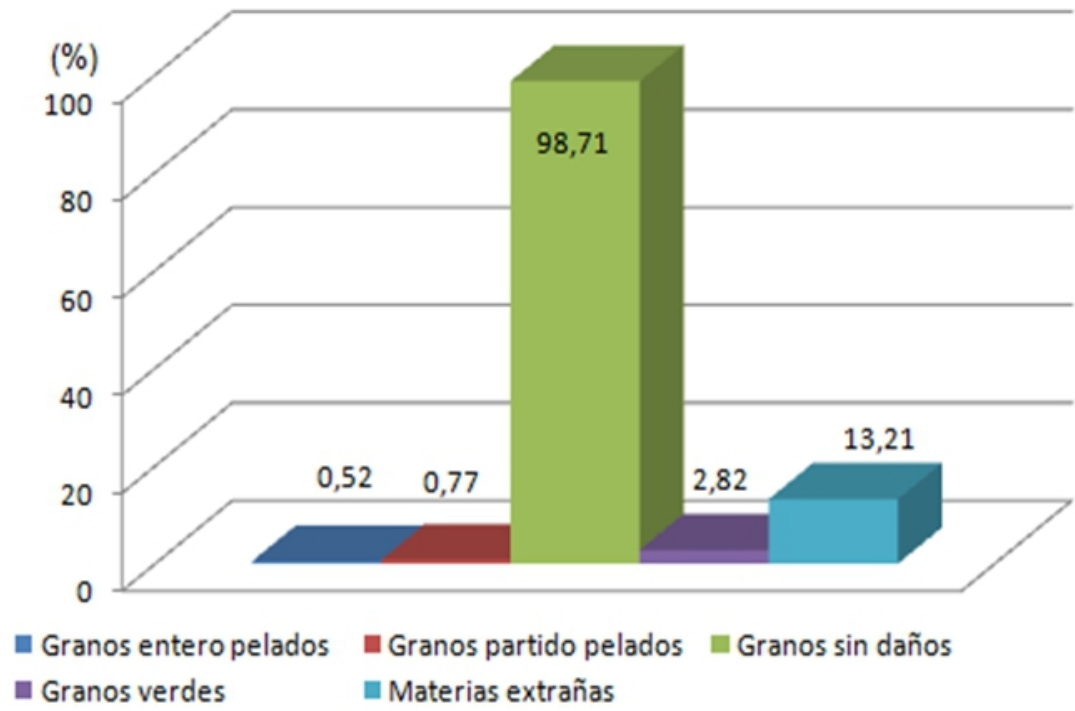

Figura 8 . Porcentaje de materias extrañas en la masa cosechada

Otra de las variables de mayor incidencia fue el valor de los granos verdes contenidos en la masa cosechada con valores de 2,3 a $4,5 \%$ con un 
promedio de 2,82, estando por encima del valor permitido en 0,3 a 2,5; un aspecto que influyó fue el flujo de los ventiladores y la humedad del cultivo.

En cuanto a la variable vegetales y tallo, se comportó en el rango, pues los resultados oscilaron entre 1,5 a $2 \%$, siendo este último el valor máximo que plantea la industria.

No obstante, se pudo comprobar con las pruebas realizadas en la industria, que la humedad del grano sobrepasaba el límite superior de humedad, lo que provoca una mala calidad de los sistemas de limpieza y como consecuencias mayor cantidad de materias extrañas en la masa cosechada, proporcionando dificultades en la limpieza del producto final.

Al realizar un análisis global de la calidad de la masa cosechada se puede observar en la figura 9, que el $87 \%$ de la masa cosechada, los granos no presentaron ningún tipo de daño mecánico, y las impurezas sobrepasan los valores establecidos para la industria con un valor del $13 \%$, superando el valor de la industria en un $30 \%$, lo cual se considera de mala calidad el producto cosechado.

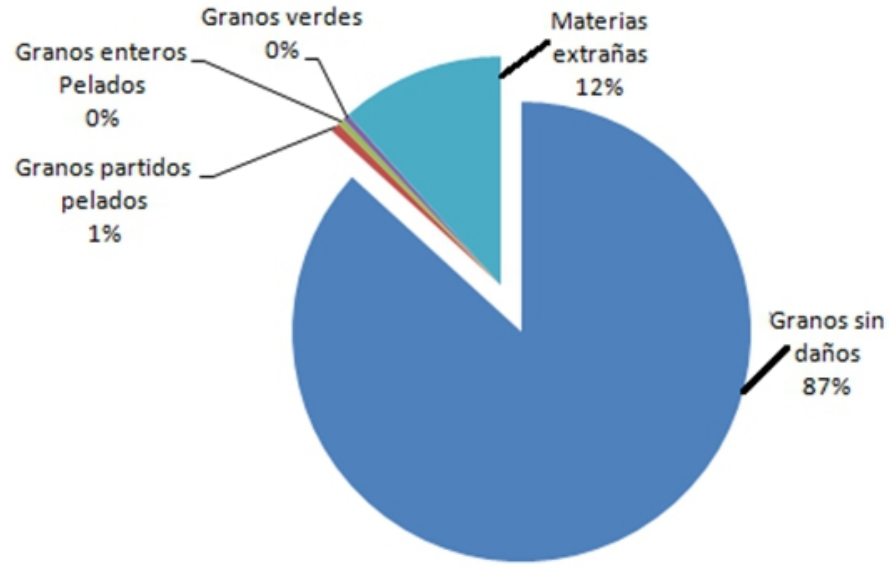

Figura 9. Calidad de la masa cosechada

\section{Conclusiones}

Las pérdidas de la cosechadora determinadas en el estudio alcanzaron $5,98 \%$, encontrándose por encima de los parámetros establecidos por el fabricante $5 \%$. Existen diferencias significativas entre las pérdidas detectadas en los diferentes sistemas de la combinada, con mejor comportamiento en la zaranda con $1,62 \%$ seguido del sacudidor con $2,03 \%$.

Las pérdidas de precosecha alcanzaron un 3,5\%, muy superior a lo establecido por la industria $1 \%$. Los contenidos de impurezas encontrados en la masa cosechada fueron de $13 \%$, superior al establecido en un $30 \%$.

Por concepto de pérdidas totales de la cosechadora se determinó 23 $920 \mathrm{t}$ de arroz limpio, lo que representa el arroz consumo de 322222 persona en un año. 


\section{Agradecimiento}

A la Granja Agrícola Arrocera "Bartolomé Masó Márquez" de la Unidad Empresarial de Base "Fernando Echenique por facilitar la maquinaria y la información básica para la ejecución de la presente investigación.

\section{References:}

1. Alonso, A.; Machado, A.; Dos Reis, R.; Machado, C; Tillmann, D; Taescher, C. (1999). Harvest loss in the irrigated rice crop with the combine operating with two kinds of header. Brazilian Congress of Agricultural Engineering. Brasil, p. 28.

2. Andrews, S.; Siebenmorgen, T.; Vories, E.; Loewer, D.; Mauromoustakos, A. (1993). Effects of Combine Operating Parameters on Harvest Loss and Quality in Rice. Transactions of the ASAE, 36(6): 1599-1607, 1993, ISSN: 2151-0059, Doi: 10.13031/2013.28501.

3. Aristizabal, Q.; Baquero, P.; Rey, V.; Acosta, N. (1999). El entorchamiento del arroz en suelos de los Llanos Orientales de Colombia. Información Técnica, 3(23). Recuperado de: http://bibliotecadigital.agronet.gov.co/bitstream/11348/6415/2/El\%20 entorchamiento\%20del\%20arroz.pdf. [Consulta: 30 de agosto de 2018].

4. Carballosa, A; Gaskins, B; Valera, C; Maciel. A. (2014). Determinación de las propiedades elásticas del cultivo de guayaba de la variedad Calvillo Medio Chino (Psidium guajava L.). Revista Ciencias Técnicas Agropecuarias, ISSN -1010-2760, RNPS-0111, Vol. 23, No. 2 (abril-mayo-junio). pp. 12-17.

5. Chaudhary R., Nanda, J. Tran, D. (2003). Guía para identificar las limitaciones de campo en la producción de arroz. Comisión internacional del arroz organización de las naciones unidas para la agricultura y la alimentación. Recuperado de: http://www.fao.org/docrep/ 006/y2778s/y2778s00.htm\#. [Consulta: 29 de septiembre de 2018].

6. Ferreira, D.; Ferreira, O.; Alonso, A.; Bley, H. (2001). Grain Loss Monitoring During all Harvest Season (Gathering and Processing Losses), in the Irrigated Rice Crop, and its Results in Reduction Losses Due to Immediate Adjustments in the Combines. No. 011075. Inst. ASAE. Annual International Meeting, Sacramento. California. USA.

7. González, T. (2017). Informe anual, Arroz. Ed. Instituto de Investigaciones del Arroz. La Habana. Cuba. Folleto.

8. INTA (2006). Nuevos consejos técnicos y reducción de tolerancias de pérdida para el 2007. Recuperado de: 
http://www.cosechaypostcosecha.org/data/gacetillas/2007/20070126e ficienciaMaiz.asp. [Consulta: 5 de septiembre de 2018].

9. INTA (2015). Instituto Nacional de Tecnología Agropecuaria. Control de pérdidas en cosecha de arroz. Recuperado de: http://www.cosechay postcosecha.org. [Consulta: 15 de septiembre de 2018].

10. Matos, R.; García, C.; González, G. (2010). Evaluación técnica y de explotación de las cosechadoras de caña Case-7 000. Revista Ciencias Técnicas Agropecuarias. 19(4): 6-9. ISSN: 2071-0054.

11. Miranda, A; Iglesias, C; Herrera, E; Abraham, N; Castells, S. (2010). Determinación de los principales parámetros de calidad que afectan la cosecha mecanizada de arroz Revista Ciencias Técnicas Agropecuarias, Vol. 19, No. 4.

12. Miranda, C.; Iglesias, C.; Rivero, F.; Falcón, C. (2006). Análisis de la calidad del arroz cosechado con las cosechadoras New Holland L520 e Ideal International 9075, Revista Ciencias Técnicas Agropecuarias, 15(3): 56-59, 2006, ISSN: 2071-0054.

13. Morejón, Y. (2015). Determinación de la composición racional del complejo cosecha-transporte del arroz con la aplicación de la teoría del servicio masivo en la empresa agroindustrial de granos "Los Palacios". Universidad Agraria de La Habana. Tesis de Doctorado. Mayabeque, Cuba.

14. Normas Brasileiras. (1987). Colhedora autopropelida de grãos. Determinação das características técnicas e de desempenho. NBR 9740, $\quad$ pp. $14 . \quad$ Recuperado de: https://www.target.com.br/produtos/normas-tecnicas/35179/nbr9740colhedora-autopropelida-de-graos-determinacao-das-caracteristicastecnicas-e-de-desempenho. [Consulta: 30 de agosto de 2018].

15. NRAG-910:88. (1988). Arroz cáscara húmedo. Determinación de materias extrañas.

16. NRAG-920: 88. (1988). Arroz cáscara húmedo. Determinación de cáscaras, pajas y granos.

17. Platero, A. (1992). Determinación de pérdidas en la cosecha del arroz durante las zafras 1986/87 y 1987/88. Arrozal 33, SER. Boletín de Divulgación, no. 18. Ed. INIA, Uruguay. ISBN: 9974-38-111-8.

18. Pozzolo, O. y Pirovani, A. (1990). Determinación de pérdidas en la cosecha de arroz. INTA. EEA C. Uruguay. Información para el productor $\mathrm{N}^{\mathrm{o}} 8$.

19. Pozzolo, O.; Ferrari, H.; Hidalgo, R.; Mirón, M. (2006). Control de pérdidas en cosecha de arroz. [en línea], Ed. PRECOP - INTA - EEA Manfredi, Córdoba, Argentina. Recuperado de: http://www.cosechaypostcosecha.org/data/articulos/cosecha/perdidas CosechaArroz.asp. [Consulta: 5 de septiembre de 2018]. 
20. Ramos, S. (2018). Determinación de las pérdidas de arroz cáscara, en cosecha manual vs cosecha mecanizada en el fundo Paredones, Valle del Bajo Piura. Tesis en opción al título de Ingeniero Agrícola. Universidad Nacional de Piura. Facultad de Agronomía. Escuela Profesional de Ingeniería Agrícola. Recuperado de: [http://repositorio.unp.edu.pe/bitstream/handle/UNP/1252/AGRRAM-CAL-17.pdf?sequence $=1 \&$ isAllowed $=y] \quad[$ Consulta: 3 de noviembre de 2018].

21. Torres-Sotolongo D, Martínez, A. (2017). Caracterización de las pérdidas achacables a la plataforma de corte-recepción durante la cosecha mecanizada del arroz. Revista Ingeniería Agrícola, ISSN2306-1545, E-ISSN-2227-8761, Vol. 7, No. 4 (octubre-noviembrediciembre), pp. 31-36. 